\title{
AÇÕES EDUCATIVAS DESENVOLVIDAS POR ENFERMEIROS BRASILEIROS COM ADOLESCENTES VULNERÁVEIS ÀS DST/AIDS
}

\author{
EDUCATIONAL ACTIONS DEVELOPED BY BRAZILIAN NURSES WITH \\ ADOLESCENTS VULNERABLE TO STD/AIDS
}

\section{ACCIONES EDUCATIVAS DESARROLLADAS POR ENFERMEROS BRASILEÑOS CON ADOLESCENTES VULNERABLES A LAS DTS/SIDA}

\author{
IZAILDO TAVARES LUNA* \\ KelanNe Lima Da SiLVA ${ }^{* *}$ \\ Fernanda Lima Aragão Dias ${ }^{* * *}$ \\ Marta Maria Costa Freitas ${ }^{* * * *}$ \\ Neiva Francenely Cunha Vieira ${ }^{* * * * *}$ \\ Patrícia Neyva da Costa Pinheiro ${ }^{* * * * *}$
}

\begin{abstract}
RESUMO
A pesquisa objetivou caracterizar os trabalhos produzidos e sintetizar a contribuição destes para o enfoque das ações educativas desenvolvidas por enfermeiros brasileiros com adolescentes em situação de vulnerabilidade às DST/Aids por meio da revisão integrativa de literatura. Para a busca dos artigos utilizaram-se os descritores indexados em Descritores em Ciências da Saúde e Medical Subject Heading, sendo selecionados 6 artigos. A síntese dos estudos evidenciou que o trabalho em grupo favorece a integração dos sujeitos envolvidos no processo, a troca de experiências e informações e a conscientização a partir do uso de metodologias como a de Paulo Freire, e o pensamento sistemático do Grupo Operativo que sensibiliza o sujeito à mudança de comportamento e, consequentemente, à melhoria da qualidade de vida. Ao final, a revisão integrativa permitiu visualizar a necessidade de os adolescentes conhecerem as maneiras de se prevenir das DST/AIDS e desenvolverem a sexualidade de forma segura, como também a aquisição de conhecimento acerca da importância das pesquisas que priorizam as ações educativas do enfermeiro, já que este deve exercer o seu papel de educador em saúde em todos os locais de atuação.
\end{abstract}

Palavras chave: Educação em saúde, saúde do adolescente, vulnerabilidade, doenças sexualmente transmissíveis, síndrome de imunodeficiência adquirida.

\footnotetext{
ABSTRACT

The research aimed to characterize the publications produced and synthesize their contribution to the orientation of educational actions developed by Brazilian nurses with adolescents in vulnerable situation to STD/AIDS through integrative review of literature. The searching for articles used descriptors indexed to

*Enfermeiro, professor substituto do Departamento de Enfermagem da Universidade Federal do Ceará, E-mail: izaildo@ yahoo.com.br

${ }^{* *}$ Enfermeira, Email: lany_lds@hotmail.com

${ }^{* * *}$ Enfermeira, Email: ferlimara@yahoo.com.br

${ }^{* * * *}$ Enfermeira, Email: martamariafreitas@hotmail.com

${ }^{* * * * *}$ Enfermeira, diretora da Faculdade de Farmácia Odontologia e Enfermagem da UFC, Fortaleza, Ceará, Brasil. Email: neivafrancenely@hotmail.com

${ }_{* * * * * *}$ Enfermeira, docente do Programa de Pós-Graduação em Enfermagem da UFC, Fortaleza, Ceará, Brasil. Email: neyva. pinheiro@yahoo.com.br
} 
Health Sciences descriptors and Medical Subject Heading, so six articles were selected. The synthesis of the studies showed that the group work promotes the integration of the individuals involved in the process, the exchange of experiences and information, and the awareness based on the use of methodologies like Paulo Freire's and the systematic thought of Operative Group that sensitizes the individual to the change of behavior and, consequently, to improve the quality of life. The integrative review allowed us to visualize the adolescents' need of knowing the ways to prevent STD/AIDS and safely develop sexuality, as well as acquiring knowledge concerning the importance of the research to prioritize the nurses' educational actions, since nurses should perform their role as health educator in all the places of professional performance.

Key words: Health education, adolescent health, vulnerability, sexually transmitted diseases, acquired immunodeficiency syndrome.

\section{RESUMEN}

El objetivo de la investigación fue caracterizar los trabajos producidos y sintetizar su contribución a la orientación de las actividades educativas desarrolladas por los enfermeros brasileños con adolescentes en situación de vulnerabilidad a las DTS/SIDA a través de la revisión integradora. Para buscar artículos se utilizaron los descriptores indexados a Descriptores en Ciencias de la Salud y Medical Subject Heading; seis artículos fueron seleccionados. Un resumen de los estudios señaló que el trabajo en grupo promueve la integración de las personas que participan en el proceso, el intercambio de experiencias e información y la toma de conciencia a través de la utilización de metodologías como la de Paulo Freire y el pensamiento sistemático del Grupo Operativo que sensibiliza al sujeto al cambio de comportamiento y, en consecuencia, a la mejoría de la calidad de vida. Por último, la revisión integradora permitió no sólo visualizar la necesidad de los adolescentes a conocer las maneras de prevenir las DTS/SIDA y el desarrollo de la sexualidad de forma segura, sino también la adquisición de conocimiento acerca de la importancia de la investigación para priorizar las actividades educativas del enfermero, ya que éste debe ejercer su papel como educador en salud en todos los lugares de la práctica.

Palabras clave: Educación en salud, salud del adolescente, vulnerabilidad, enfermedades de transmisión sexual, síndrome de inmunodeficiencia adquirida.

Fecha recepción: 06/12/10 Fecha aceptación: 23/11/11

\section{INTRODUÇÃO}

Desde a década de 1980, quando surgiram os primeiros casos de AIDS no cenário epidemiológico mundial, a prevenção das Doenças Sexualmente Transmissíveis (DSTs) e do Vírus da Imunodeficiência Adquirida (HIV) entre adolescentes tem sido um dos maiores desafios no controle dessas doenças (1).

Estimativas da Organização Mundial da Saúde (2) sinalizam que cerca de $50 \%$ das novas infecções pelo HIV estão ocorrendo na adolescência. Em todo o mundo há mais de 40 milhões de pessoas vivendo com AIDS. Entre esse total, aproximadamente, $30 \%$ se encontram na faixa etária de 15 a 24 anos.
Isso representa cerca de 12 milhões de pessoas infectadas.

No Brasil, nesses quase 30 anos de epidemia de AIDS, percebem-se mudanças significativas no que se refere à sua dinâmica da transmissão. Na atualidade, a epidemia apresenta incremento de casos entre heterossexuais, mulheres e adolescentes. O panorama assinala não mais em direção a grupos ou comportamentos específicos, mas a um agregado de variáveis, mais complexo e emaranhado do que se pensava no início da epidemia (3).

A vulnerabilidade dos adolescentes às DST/AIDS está envolta aos determinantes biológicos e aos aspectos psicológicos caracterizados pela percepção de invulnerabilidade, imortalidade e influência das relações de 
gênero, fatores estes que influenciam diretamente no risco à transmissão desses agravos à saúde (4).

Em todo o mundo o tema prevenção de DST/AIDS entre adolescentes aponta como um campo de crescente investigação dos estudos em saúde, incluindo os da área de Enfermagem, a qual tem produzido debates a fim de promover uma leitura mais fidedigna dessa realidade.

Dentre os desafios que o enfermeiro enquanto educador em saúde enfrenta para a redução das taxas infecção de DST/AIDS entre os adolescentes, tem-se o empoderamento desses sujeitos ao comportamento sexual seguro, assunto de interesse dos profissionais de Enfermagem, com vistas a minimizar os riscos dessa população às DST/AIDS (5).

Nesse contexto surge a importância da práxis do enfermeiro como educador em saúde, na medida em que sua prática esteja imbuída em competências e habilidades no âmbito da Educação em Saúde para promover em um espaço dialógico a construção da consciência crítica-reflexiva nos sujeitos visando à adoção de comportamento seguro frente às DST/AIDS. Por isso, surgiu o interesse em desenvolver esta revisão integrativa de literatura sobre as ações educativas desenvolvidas por enfermeiros brasileiros com adolescentes visando à prevenção das DST/ AIDS.

A revisão integrativa é uma ferramenta importante no processo de análise de comunicação dos resultados de pesquisas, facilitando sua utilização na prática clínica, uma vez que proporciona uma síntese do conhecimento já produzido e fornece subsídios para a melhoria da assistência à saúde, contribuindo, a partir dos resultados de pesquisas já produzidas, na elaboração e fundamentação de novos trabalhos (6). Visa oferecer, ainda, aos profissionais de diversas áreas de atuação na saúde, o acesso rápido aos resultados relevantes de pesquisas que fundamentam as condutas ou a tomada de decisão, proporcionando um saber crítico que se fundamente na redução dos obstáculos da utilização do conhecimento científico e tornando mais acessíveis os resultados das pesquisas (7).

Neste estudo a questão norteadora da revisão integrativa foi: Quais as estratégias educativas desenvolvidas por enfermeiros brasileiros para a prevenção de DST/AIDS entre os adolescentes?

Em busca de melhorias na pesquisa e no desenvolvimento das competências de Enfermagem na Educação em Saúde, este trabalho objetivou identificar os estudos produzidos por enfermeiros brasileiros sobre as ações educativas desenvolvidas com adolescentes para a prevenção de DST/AIDS.

\section{METODOLOGIA}

O estudo faz uma revisão integrativa de literatura sobre as ações educativas desenvolvidas por enfermeiros brasileiros com adolescentes em situação de vulnerabilidade às DST/AIDS.

A revisão integrativa é definida como a construção de uma análise ampla de literatura, contribuindo para as discussões sobre métodos e resultados de pesquisas, assim como reflexões sobre a realização de futuros estudos. Esse método de pesquisa permite a síntese de estudos publicados e possibilita conclusões gerais a respeito de uma particular área de estudo (8).

Para a operacionalização dessa revisão integrativa utilizamos as seguintes etapas: identificação da questão da pesquisa para reconhecimento dos descritores; busca de literatura conforme os critérios de inclusão e exclusão; categorização dos artigos a partir do instrumento da coleta de dados, que definiu as informações a serem extraídas dos estudos selecionados; avaliação dos estudos pela análise minuciosa dos principais dados que foram sintetizados em tabelas; interpretação dos dados pelas comparações realiza- 
das entre os resultados dos dados coletados de cada artigo; e síntese do conhecimento evidenciado em todos os artigos analisados $(9,10)$.

O levantamento bibliográfico foi realizado de maio a setembro de 2010 nas bases de dados Literatura Latino-Americana em Ciências da Saúde (LILACS) e National Library of Medicine (MEDLINE). Para o levantamento dos artigos utilizamos os descritores indexados nos Descritores em Ciência da Saúde (DeCS): Educação em Saúde; Adolescente; DST e no Medical Subjetc Headings (MeSH): Educação em Saúde; Adolescente e AIDS, a partir da Biblioteca Virtual em Saúde (BVS).

Os critérios de inclusão dos artigos foram: artigo completo, disponível eletronicamente nas referidas bases de dados, com data de publicação entre os anos de 2000 e 2010, nos idiomas português, espanhol ou inglês. Os critérios de exclusão foram: artigo repetido que estivesse em mais de uma base de dados e que não contemplassem o objetivo do estudo.

$\mathrm{Na}$ busca inicial, utilizando os descritores Educação em Saúde, Adolescente e DST, foram encontrados 167 artigos. Destes 36 na base LILACS e 131 artigos na base MEDLINE. Por meio da busca com os descritores Educação em Saúde, Adolescente e AIDS foram encontrados 155 artigos, dos quais 25 na base LILACS e 130 na MEDLINE. Através da busca por artigo completo foram excluídos 126, restando 196 artigos. No entanto, apenas 12 atenderam aos critérios de inclusão e exclusão, os quais foram lidos na íntegra; no entanto, destes apenas seis responderam à questão norteadora e fizeram parte da presente revisão.

A coleta de informações ocorreu a partir de um instrumento adaptado (11) ao tema do estudo, sendo preenchido com as seguintes informações: título do artigo, ano de publicação, qualis e título do periódico, objetivos, delineamento, referencial metodológico, resultados e conclusões, bem como as temáticas desenvolvidas nas ações educativas e o papel do enfermeiro nas estratégias educativas.

Os artigos encontrados foram apresentados conforme a ordem de localização, e os dados organizados a partir de informações extraídas das publicações selecionadas. Neste sentido procedemos à apresentação dos resultados e discussão das informações de forma descritiva e por meio da construção de quadros contendo título do estudo e do periódico; ano de publicação; sujeitos do estudo; objetivos do estudo; resultados e conclusões do estudo.

\section{RESULTADOS}

Os resultados são apresentados pela ordem das questões do formulário da coleta de dados. Fizeram parte da pesquisa cinco periódicos descritos a seguir: Revista Brasileira de Enfermagem; Revista Latino-Americana de Enfermagem; Escola Anna Nery Revista de Enfermagem; DST - Jornal Brasileiro de Doenças Sexualmente Transmissíveis; Revista Enfermagem UERJ.

A análise dos estudos que compuseram esta revisão evidenciou que um dos periódicos apresenta qualis A2, quatro com qualis B1 e um classificado em B3. Com estes dados percebe-se que a maioria das pesquisas que serviram para a construção desta síntese foi publicada em revistas bem pontuadas na classificação das bases bibliográficas internacionais, o que demonstra a qualidade dos periódicos analisados neste estudo.

A caracterização dos seis artigos incluídos nesta revisão integrativa mostrou que a maioria foi publicada após o ano de 2007, à exceção de apenas um, que foi publicado em 2001. Todos foram provenientes de estudos realizados por enfermeiros brasileiros vinculados aos departamentos de Enfermagem de universidades públicas do Brasil.

Com relação à origem das publicações e 
Tabela 1. Estudos incluídos na revisão integrativa sobre as ações educativas desenvolvidas por enfermeiros brasileiros com adolescentes vulneráveis as DST/AIDS. Fortaleza, CE, Brasil, 2010 .

\begin{tabular}{|c|c|c|c|c|}
\hline Qualis $^{\star}$ & Periódico/Ano & Sujeitos do estudo & $\begin{array}{l}\text { Referencial } \\
\text { metodológico }\end{array}$ & $\begin{array}{l}\text { Delineamento do } \\
\text { estudo }\end{array}$ \\
\hline B1 & $\begin{array}{l}\text { Rev Bras Enferm./ } \\
2007\end{array}$ & $\begin{array}{l}\text { Adolescentes de baixa } \\
\text { renda do Município } \\
\text { de Aparecida de Goi- } \\
\text { ânia/GO. }\end{array}$ & $\begin{array}{l}\text { Metodologia partici- } \\
\text { pativa de Paulo Freire }\end{array}$ & Qualitativo \\
\hline A2 & $\begin{array}{l}\text { Revista Latino-Am } \\
\text { Enfermagem./2007 }\end{array}$ & $\begin{array}{l}\text { Um grupo de adoles- } \\
\text { centes que vivem em } \\
\text { um contexto de po- } \\
\text { breza. }\end{array}$ & $\begin{array}{l}\text { Pensamento Sistêmi- } \\
\text { co e o Grupo Opera- } \\
\text { tivo }\end{array}$ & Qualitativo \\
\hline B1 & $\begin{array}{l}\text { Esc Anna Nery Rev } \\
\text { Enferm./ } 2008\end{array}$ & $\begin{array}{l}\text { Um grupo de } 10 \text { me- } \\
\text { ninas, entre } 14 \text { e } 16 \\
\text { anos, que estavam } \\
\text { matriculadas na esco- } \\
\text { la onde o estudo foi } \\
\text { realizado. }\end{array}$ & Círculo de Cultura & Qualitativo \\
\hline B3 & $\begin{array}{l}\text { DST - J Bras Do- } \\
\text { enças Sex Transm./ } \\
2001\end{array}$ & $\begin{array}{l}\text { Mulheres adolescentes } \\
\text { que estudam no turno } \\
\text { da noite, na faixa etá- } \\
\text { ria de } 13 \text { a } 19 \text { anos. }\end{array}$ & Pesquisa participante & Qualitativo \\
\hline B1 & $\begin{array}{l}\text { Revista de Enferma- } \\
\text { gem UERJ/ } 2010\end{array}$ & $\begin{array}{l}\text { Adolescentes e jovens } \\
\text { com idades entre } 16 \text { e } \\
24 \text { anos, de ambos os } \\
\text { sexos, em situação de } \\
\text { vulnerabilidade so- } \\
\text { cial, oriundos de co- } \\
\text { munidades empobre- } \\
\text { cidas de Florianópolis }\end{array}$ & Pesquisa-Ação & Qualitativo \\
\hline B1 & $\begin{array}{l}\text { Revista de Enferma- } \\
\text { gem UERJ / } 2010\end{array}$ & $\begin{array}{l}\text { Grupo de } 25 \text { adoles- } \\
\text { centes de } 14 \text { a } 20 \text { anos } \\
\text { de idade que estu- } \\
\text { davam numa escola } \\
\text { pública da cidade de } \\
\text { Fortaleza-CE, no } 1^{\circ} \\
\text { ano do ensino médio. }\end{array}$ & Pesquisa-Ação & Qualitativo \\
\hline
\end{tabular}

* Qualis: forma utilizada pela CAPES (Coordenação de Aperfeiçoamento de Pessoal de Nível Superior) para classificar os periódicos segundo o valor do fator de impacto ou estratificação indicativa de qualidade. Assim, os periódicos são classificados em ordem decrescente de estrato de qualidade em A1 (o mais elevado), A2, B1, B2, B3, B4, B5 e C (peso zero). 
Tabela 2. Estudos incluídos na revisão integrativa, segundo título, autores, objetivo e método, resultados e discussão, recomendações e conclusões. Fortaleza, CE, Brasil, 2010.

\begin{tabular}{|c|c|c|c|c|}
\hline Título do estudo & Autores & Objetivos do estudo & Resultados & Conclusões \\
\hline $\begin{array}{l}\text { Programa educa- } \\
\text { tivo sobre sexuali- } \\
\text { dade e DST: relato } \\
\text { de experiência } \\
\text { com grupo de } \\
\text { adolescentes (12) }\end{array}$ & $\begin{array}{l}\text { Souza, MM; Bru- } \\
\text { nini, S; Almeida, } \\
\text { NAM; Munari, DB }\end{array}$ & $\begin{array}{l}\text { Relatar a experiência } \\
\text { com grupo de adoles- } \\
\text { centes através de ofi- } \\
\text { cinas vivenciais sobre } \\
\text { educação sexual. }\end{array}$ & $\begin{array}{l}\text { Possibilitou a interação } \\
\text { mútua e os objetivos } \\
\text { entre os coordenado- } \\
\text { res e os grupos de ado- } \\
\text { lescentes. Destacou a } \\
\text { importância da trans- } \\
\text { formação da realidade, } \\
\text { bem como da mudan- } \\
\text { ça de comportamento } \\
\text { para hábitos saudáveis e } \\
\text { atitudes positivas. }\end{array}$ & $\begin{array}{l}\text { Possibilitou o alcance } \\
\text { dos objetivos propos- } \\
\text { tos, considerando que } \\
\text { esse modelo é funda- } \\
\text { mentado no diálogo } \\
\text { aberto e reflexivo e que } \\
\text { o educando parte de } \\
\text { sua própria realidade } \\
\text { para interagir, trocar } \\
\text { experiências e adquirir } \\
\text { novos conhecimentos. }\end{array}$ \\
\hline $\begin{array}{l}\text { Conversando so- } \\
\text { bre sexualidade, } \\
\text { IST e aids com } \\
\text { adolescentes po- } \\
\text { bres (13) }\end{array}$ & $\begin{array}{l}\text { Murakami, JK; Pe- } \\
\text { trilli Filho, FJ; Telles } \\
\text { Filho, PC }\end{array}$ & $\begin{array}{l}\text { Relatar a experiência } \\
\text { de uma ação educati- } \\
\text { va sobre sexualidade/ } \\
\text { IST/Aids junto a um } \\
\text { grupo de adolescentes } \\
\text { em contexto de po- } \\
\text { breza. }\end{array}$ & $\begin{array}{l}\text { Grupo como espaço } \\
\text { de construção sistêmi- } \\
\text { ca e circular explici- } \\
\text { tou como ele pode ser } \\
\text { inspirador e revelador } \\
\text { para o trabalho do en- } \\
\text { fermeiro, uma vez que } \\
\text { permitiu a emergência } \\
\text { de uma prática conver- } \\
\text { gente com o contexto } \\
\text { sociocultural em ques- } \\
\text { tão. }\end{array}$ & $\begin{array}{l}\text { Avançar em direção a } \\
\text { uma prática afetiva e } \\
\text { dialógica em seu con- } \\
\text { texto de trabalho. }\end{array}$ \\
\hline $\begin{array}{l}\text { Ação educativa } \\
\text { do enfermeiro na } \\
\text { prevenção de do- } \\
\text { enças sexualmen- } \\
\text { te transmissíveis: } \\
\text { uma investigação } \\
\text { a partir das ado- } \\
\text { lescentes (14) }\end{array}$ & $\begin{array}{l}\text { Beserra, EP; Pinhei- } \\
\text { ro, PNC; Barroso, } \\
\text { MGT }\end{array}$ & $\begin{array}{l}\text { Investigar a sexualida- } \\
\text { de das adolescentes a } \\
\text { partir da ação educa- } \\
\text { tiva do enfermeiro na } \\
\text { prevenção de doenças } \\
\text { sexualmente trans- } \\
\text { missíveis. }\end{array}$ & $\begin{array}{l}\text { As meninas associam } \\
\text { o sexo à sexualidade de } \\
\text { forma predominante e } \\
\text { que tinham pouca com- } \\
\text { preensão das vulnera- } \\
\text { bilidades que estavam } \\
\text { expostas numa prática } \\
\text { sexual desprotegida. } \\
\text { Observou-se como as } \\
\text { adolescentes percebem } \\
\text { a família, a escola e os } \\
\text { amigos acerca da sexu- } \\
\text { alidade na juventude, } \\
\text { constatando-se a neces- } \\
\text { sidade de intervir nesse } \\
\text { grupo a partir de suas } \\
\text { necessidades específi- } \\
\text { cas. Evidenciou-se que a } \\
\text { execução do Círculo de } \\
\text { Cultura permitiu que } \\
\text { as meninas discutissem } \\
\text { sobre diversos temas } \\
\text { que englobavam sua se- } \\
\text { xualidade. }\end{array}$ & $\begin{array}{l}\text { Constatou-se com esse } \\
\text { estudo que são neces- } \\
\text { sárias ações de educa- } \\
\text { ção em saúde, como } \\
\text { o Círculo de Cultura, } \\
\text { que propiciam o jovem } \\
\text { expor suas dúvidas } \\
\text { e conhecer os meios } \\
\text { de prevenção, capa- } \\
\text { citando-o a repensar } \\
\text { condutas, favorecendo } \\
\text { uma melhor qualida- } \\
\text { de de vida e sendo um } \\
\text { método eficaz para a } \\
\text { aprendizagem. Nesse } \\
\text { contexto, a Enferma- } \\
\text { gem preocupa-se com } \\
\text { as ações de educação } \\
\text { em saúde, podendo } \\
\text { executar essa técnica } \\
\text { para trabalhar com as } \\
\text { jovens em diferentes } \\
\text { setores e segmentos } \\
\text { sociais com a meta de } \\
\text { isentá-las de riscos. }\end{array}$ \\
\hline
\end{tabular}


Continuação Tabela 2.

\begin{tabular}{|c|c|c|c|c|}
\hline $\begin{array}{l}\text { Sexualidade, DST/ } \\
\text { AIDS e adoles- } \\
\text { cência: não quero } \\
\text { falar, tenho vergo- } \\
\text { nha (15) }\end{array}$ & $\begin{array}{l}\text { Vieira, NFC; Paiva, } \\
\text { TC; Sherlock, MSM }\end{array}$ & $\begin{array}{l}\text { Conhecer as percep- } \\
\text { ções da adolescente, } \\
\text { acerca da sexualidade, } \\
\text { em tempos de aids, } \\
\text { para que se possa pro- } \\
\text { mover estratégias de } \\
\text { educação em saúde } \\
\text { que atenda as neces- } \\
\text { sidades dessa clien- } \\
\text { tela nas tomadas de } \\
\text { decisão que envolvam } \\
\text { o domínio do corpo } \\
\text { biológico, psicossocial } \\
\text { e afetivo, promovendo } \\
\text { comportamentos } \\
\text { sexuais seguros. }\end{array}$ & $\begin{array}{l}\text { As adolescentes ma- } \\
\text { nifestaram dúvidas, } \\
\text { principalmente acerca } \\
\text { dos métodos anticon- } \\
\text { ceptivos, o medo e o } \\
\text { constrangimento de fa- } \\
\text { lar sobre sexo e, ainda, } \\
\text { expectativas em relação } \\
\text { ao comportamento se- } \\
\text { xual futuro. }\end{array}$ & $\begin{array}{l}\text { Concluiu-se que sexo } \\
\text { e sexualidade são um } \\
\text { tema cercado por ta- } \\
\text { bus, o que dificulta o } \\
\text { trabalho. Apesar de } \\
\text { elas terem informações } \\
\text { acerca das medidas de } \\
\text { prevenção das DST/ } \\
\text { AIDS, esse conheci- } \\
\text { mento ainda não pa- } \\
\text { rece suficiente para } \\
\text { assegurar o compor- } \\
\text { tamento sexual livre } \\
\text { de riscos. É necessário } \\
\text { o incentivo a ações de } \\
\text { educação em saúde } \\
\text { voltadas para o público } \\
\text { em questão. }\end{array}$ \\
\hline $\begin{array}{l}\text { Sexualidade, do- } \\
\text { enças sexualmen- } \\
\text { te transmissíveis } \\
\text { e contracepção: } \\
\text { atuação da enfer- } \\
\text { magem com jo- } \\
\text { vens de periferia } \\
\text { (16) }\end{array}$ & $\begin{array}{l}\text { Koerich, MS; Ba- } \\
\text { ggio, MA; Backes, } \\
\text { MTS; Backes, DS; } \\
\text { Carvalho, JN; Mei- } \\
\text { relles, BHS; Erd- } \\
\text { mann, AL }\end{array}$ & $\begin{array}{l}\text { Discutir sexualidade, } \\
\text { DST e contracepção a } \\
\text { partir da concepção de } \\
\text { jovens de comunidades } \\
\text { periféricas de Floria- } \\
\text { nópolis, apresentando } \\
\text { a importância da atu- } \\
\text { ação da enfermagem } \\
\text { junto a essa população. }\end{array}$ & $\begin{array}{l}\text { Os adolescentes ma- } \\
\text { nifestaram interesse } \\
\text { por temas referentes } \\
\text { à estrutura biológi- } \\
\text { ca e à saúde sexual, } \\
\text { apresentando diversos } \\
\text { questionamentos re- } \\
\text { lacionados a doenças } \\
\text { dos órgãos reprodu- } \\
\text { tivos. Apresentaram } \\
\text { dúvidas, curiosidades e } \\
\text { falhas em suas concep- } \\
\text { ções sobre os conceitos } \\
\text { proteção contra DST/ } \\
\text { AIDS e anticoncepção. }\end{array}$ & $\begin{array}{l}\text { A AIDS é a DST mais } \\
\text { conhecida. É, portanto, } \\
\text { fundamental que os } \\
\text { profissionais da saúde, } \\
\text { especialmente a en- } \\
\text { fermagem, busquem } \\
\text { atuar nesses espaços } \\
\text { sociais vulneráveis. } \\
\text { Torna-se imprescin- } \\
\text { dível a expansão das } \\
\text { ações da enfermagem } \\
\text { para atuar efetivamen- } \\
\text { te com os adolescentes } \\
\text { e jovens das periferias, } \\
\text { especialmente no que } \\
\text { se refere a assuntos } \\
\text { relacionados à sexu- } \\
\text { alidade, DST/AIDS e } \\
\text { contracepção. }\end{array}$ \\
\hline $\begin{array}{l}\text { Riscos e vulnera- } \\
\text { bilidades relacio- } \\
\text { nados à sexualida- } \\
\text { de na adolescência } \\
\text { (17) }\end{array}$ & $\begin{array}{ll}\text { Dias, FLA; } & \text { Silva, } \\
\text { KL; Vieira, } & \text { NFC; } \\
\text { Pinheiro, } & \text { PNC; } \\
\text { Maia, CC } & \end{array}$ & $\begin{array}{l}\text { Relatar os efeitos das } \\
\text { ações de Educação } \\
\text { em Saúde junto à es- } \\
\text { cola que visaram à } \\
\text { conscientização dos } \\
\text { adolescentes acerca da } \\
\text { realização de medidas } \\
\text { preventivas para DSTs } \\
\text { e gravidez não plane- } \\
\text { jada. }\end{array}$ & $\begin{array}{l}\text { A camisinha é o meio } \\
\text { mais conhecido pelos } \\
\text { adolescentes, apesar } \\
\text { de ser utilizada erro- } \\
\text { neamente e de modo } \\
\text { irregular. Muitos dos } \\
\text { adolescentes do es- } \\
\text { tudo não conhecem } \\
\text { o próprio corpo e se } \\
\text { mostraram incapazes } \\
\text { de reconhecer os sinto- } \\
\text { mas de uma DST e as } \\
\text { formas de transmissão } \\
\text { da AIDS. }\end{array}$ & $\begin{array}{l}\text { Os adolescentes rejei- } \\
\text { tam qualquer forma } \\
\text { de conhecimento a } \\
\text { eles imposta. Assim, os } \\
\text { profissionais que de- } \\
\text { senvolvem grupos de } \\
\text { discussão envolvendo } \\
\text { adolescentes precisam } \\
\text { acolher e envolver es- } \\
\text { ses sujeitos de forma } \\
\text { dinâmica, possibilitan- } \\
\text { do ser o conhecimento } \\
\text { constituído de troca de } \\
\text { informações, pois não } \\
\text { basta apenas informar. }\end{array}$ \\
\hline
\end{tabular}


instituições-sede dos autores, predominaram os estudos realizados por pesquisadores vinculados ao Departamento de Enfermagem da Universidade Federal do Ceará, com três estudos publicados, seguidos pelo Departamento de Enfermagem da Universidade do Estado de Goiás, o Departamento de Enfermagem da Universidade do Estado de São Paulo e pelo Departamento de Patologia da Universidade Federal de Santa Catarina, com um artigo publicado cada.

No que diz respeito aos sujeitos envolvidos nos estudos analisados, tivemos o seguinte: as pesquisas foram realizadas com adolescentes e jovens de baixa renda que vivem em um contexto de pobreza e se apresentam em situação de vulnerabilidade social, com faixa etária de 13 a 24 anos, de ambos os sexos, e estudantes de escolas públicas das regiões onde os estudos se desenvolveram.

Acerca dos referenciais metodológicos que fundamentaram as ações educativas desenvolvidas nos estudos selecionados para a elaboração desta revisão, tivemos: dois estudos utilizaram a Pesquisa-ação, um estudo usou a metodologia participativa de Paulo Freire, outro utilizou o Pensamento Sistêmico e o Grupo Operativo, outro estudo utilizou o referencial freireano Círculo de Cultura e um utilizou o referencial da Pesquisa participante. Houve unanimidade dos estudos com abordagem qualitativa, sendo que dois foram relatos de experiência, um estudo exploratório e descritivo, duas pesquisasação e uma pesquisa participante.

Quanto aos objetivos dos estudos, dois relataram experiências vivenciadas por enfermeiros na realização de ações educativas sobre sexualidade, DST e AIDS com grupos de adolescentes; dois se propuseram a conhecer e investigar a percepção dos adolescentes acerca da sexualidade através de oficinas educativas em saúde; um relatou os efeitos das ações de Educação em Saúde junto a adolescentes escolares, e outro estudo discutiu sexualidade, DST e contracepção por meio da percepção dos jovens de comu- nidades pobres e em situação de vulnerabilidade social.

Em relação aos resultados dos estudos, a maioria evidenciou que os adolescentes manifestaram várias dúvidas em relação à sexualidade, aos métodos anticonceptivos e, principalmente, sobre a prevenção das DST/ AIDS. Muitos dos participantes dos estudos apresentaram medo e constrangimento em falar sobre as expectativas em relação ao comportamento sexual futuro, sendo que um grande número dos adolescentes não conhece o próprio corpo, mostrando-se incapaz de reconhecer os sintomas das DST e as formas de contágio da AIDS.

Podemos inferir, ainda, por meio dos estudos, a importância do papel exercido pela família, pela escola e pelos amigos para o enfrentamento das vulnerabilidades às DST/ AIDS pelos adolescentes, constatando-se, assim, a necessidade intervenções mais efetivas direcionadas a esse grupo a partir de suas necessidades específicas.

Os estudos mostraram, também, que o desenvolvimento de atividades educativas que estimulem a troca de experiência, o compartilhamento de conhecimentos entre os integrantes, a discussão de temáticas de interesse desse público e que sejam contextualizadas de acordo com o meio socioeconômico e cultural onde estão inseridos promove a construção de um espaço sistêmico e circular. $\mathrm{O}$ trabalho do enfermeiro como educador em saúde se apresenta de forma inspiradora e reveladora, uma vez que permite a emergência de uma prática convergente com o contexto sociocultural dos adolescentes.

As principais recomendações dos estudos acerca das ações educativas desenvolvidas por enfermeiros brasileiros com adolescente com vistas à prevenção das DST/AIDS foram: utilização da estratégia de grupo, uma vez que esta facilita a identificação das vulnerabilidades dos adolescentes; reforço quanto à importância da orientação sexual na adolescência; uso de ações educativas baseadas no diálogo aberto e reflexivo a partir da pró- 
pria realidade dos envolvidos no processo, favorecendo a aquisição de novos conhecimentos e atitudes.

Quanto às temáticas abordadas nas ações educativas desenvolvidas com os adolescentes, notou-se que imagem, corpo, prazer e DST/AIDS, comportamento/práticas sexuais (sexo anal, oral e vaginal), conhecimentos específicos sobre as formas de infecção, mitos e verdade acerca da sexualidade, uso do preservativo feminino/masculino, gravidez na adolescência; e sexo, sexualidade e métodos contraceptivos foram os temas mais discutidos nas estratégias educativas.

Acredita-se que a escolha desses temas norteadores das discussões durante as ações educativas fundamenta-se, principalmente, no fato de que o risco de infecção por uma DST/AIDS e a gravidez não planejada configuram-se como importantes problemas enfrentados na adolescência. Esses temas, atrelados à sexualidade, ao sexo e aos métodos anticonceptivos se apresentam como temáticas de interesse dos adolescentes.

A infecção de adolescentes e jovens pelas DST/AIDS se apresenta como um grave problema de saúde pública no Brasil e no mundo, visto que a fase da adolescência é marcada por eventos relacionados à iniciação precoce das relações sexuais, à não utilização ou uso inadequado do preservativo em todas as relações sexuais, à falta de diálogo com os pais e à ausência de acesso a fontes esclarecedoras confiáveis para realizações de indagações $(18,19)$.

No que compete aos principais papéis desempenhados pelo enfermeiro durante a execução das ações educativas com adolescentes, visualizou-se: coordenador das oficinas educativas, realizadas por meio do uso de metodologia participativa e lúdica; coordenador do Grupo Operativo, articulador e observador participante das estratégias educativas, facilitador do Círculo de Cultura; moderador do grupo focal; incentivador da construção da conscientização e do empoderamento coletivo.
O enfermeiro ao desenvolver diferentes papéis nas ações educativas promove no grupo uma discussão de forma dinâmica que envolve os participantes e propicia a estes a exposição das suas dúvidas em ambiente de acolhimento e envolvimento mútuo, permitindo a construção coletiva do conhecimento por meio da troca das informações e das vivências, configurando-se um método eficaz para a aprendizagem no que se refere aos assuntos relacionados à sexualidade e à prevenção das DST/AIDS (5).

\section{DISCUSSÃO E COMENTÁRIO}

Dentre os estudos analisados, a abordagem qualitativa foi unânime, visto que se trata de uma revisão integrativa que se propôs a realizar uma síntese dos estudos que abordam ações educativas com adolescentes vulneráveis as DST/AIDS. Os resultados dos estudos selecionados são frutos da interação do grupo e decorrentes dos valores, crenças e ideias dos participantes em relação ao tema abordado. Portanto, fundamenta-se neste fato a unanimidade nesta síntese de estudos qualitativos, tendo em vista que estes estão totalmente vinculadas ao comportamento humano e seus valores, pensamentos e convicções sendo particular de cada ser, não podendo ser explicados por números e estatísticas (20).

A maioria dos estudos que compõem esta revisão apresenta como público-alvo adolescentes em situação de vulnerabilidade social. Acredita-se que a escolha por trabalhar com essa população esteja fundamentada no fato de que as variadas situações de saúde-doença às quais se expõem esses sujeitos, atreladas à precocidade do início das relações sexuais presentes na adolescência, a baixa escolaridade e a pauperização se configurem como fatores condicionantes para uma maior vulnerabilidade às DST/AIDS, necessitando de ações integradas e efetivas por parte dos pro- 
fissionais da saúde e, em especial, do enfermeiro (21).

Quanto às estratégias educativas desenvolvidas, as investigações destacaram a relevância do trabalho com grupos, pois estes favorecem a interação entre os adolescentes e os coordenadores, facilitando a troca de saberes e afetos. Resgatam, também, valores dos próprios grupos para promover a conscientização sobre os riscos às DST/AIDS e, consequentemente, favorecer a mudança de comportamento a partir das necessidades específicas de cada adolescente (22).

O trabalho em grupo envolvendo a temática da prevenção das DST/AIDS beneficia o relacionamento interpessoal, facilita a formulação de saberes a partir de uma aprendizagem compartilhada com troca de experiências baseada no contexto sociocultural dos sujeitos envolvidos na ação educativa (23).

Os artigos analisados apontam que se torna imprescindível a expansão das ações da enfermagem para atuar efetivamente com os adolescentes e jovens, pois não basta informar. O enfermeiro, ao desenvolver as atividades educativas em grupo, deve facilitar a tomada de consciência dos sujeitos envolvidos no processo educativo para promover a mudança de comportamento diante das práticas sexuais, possibilitando assim, a construção de atitude crítica e reflexiva que permita a adoção de medidas preventivas às DST/ AIDS.

Os estudos mencionam, ainda, que as estratégias voltadas para trabalhar a prevenção das DST/AIDS devem priorizar a troca de experiência, o compartilhamento de conhecimentos entre os seus integrantes e a discussão de temáticas de interesse desse público. Essas estratégias devem ser contextualizadas de acordo com o meio socioeconômico e cultural em que os sujeitos estão inseridos, além de suas necessidades específicas (24).

Com base nas propostas apresentadas nos estudos analisados envolvendo ações de Educação em Saúde com adolescentes vulneráveis às DST/AIDS, percebemos que a ação educativa desenvolvida pelo enfermeiro necessita ser planejada e implementada com utilização de diversas estratégias metodológicas para nortear uma prática efetiva.

A operacionalização de metodologias educativas de forma efetiva promove a conscientização do sujeito visando comportamentos e atitudes frente às DST/AIDS.

Com os dados apresentados infere-se que em sua maioria os estudos adotaram referenciais teóricos provenientes de metodologias emancipatórias, dialogais, autônomas e participativas. Ao utilizar essas metodologias os estudos fortaleceram o suporte para a mudança de comportamento e o estilo de vida dos adolescentes, favorecendo a promoção humana, na medida em que encorajam o sujeito ao protagonismo e ao envolvimento nas decisões de questões que dizem respeito à sua saúde (18).

Para compreender as ações educativas desenvolvidas pelo enfermeiro com ênfase na prevenção das DST/AIDS em adolescentes, faz-se necessário conhecer como a enfermagem vem desempenhando as ações de Educação em Saúde nos diversos cenários de atuação com o público adolescente, evidenciando as complexidades, os obstáculos e os inúmeros desafios enfrentados pelo enfermeiro na práxis como educador em saúde.

Diante dessa realidade o enfermeiro como educador em saúde exerce papel fundamental na construção do processo ensino-aprendizagem quando a sua prática com os grupos operativos consiste no trabalho coletivo, com o intuito de capacitar o sujeito ao seu protagonismo. Pode utilizar, ainda, oficinas educativas fundamentadas em metodologias emancipatórias como as propostas pelo educador Paulo Freire, que insere o sujeito no centro do processo de aprendizagem (25).

Ao realizar ações educativas utilizando metodologias participativas, motivadoras e lúdicas o enfermeiro demonstra como tem se empenhado na construção de novas formas de produzir saúde através de ações de caráter individual e coletivo, bem como rea- 
lizando atividades que interligam o sujeito, a família e a sociedade (26).

$O$ profissional da enfermagem, ao atuar como educador em saúde, promove o cuidado integral e criativo aprimorando a assistência de enfermagem, coloca o sujeito como participante das ações e protagonista da sua condição de vida, conscientiza-o e lhe dá subsídios para transformar a sua realidade (27).

Diante do exposto nesta revisão integrativa, foi possível conhecer os resultados provenientes do desenvolvimento de ações educativas com adolescentes em vulnerabilidade às DTS/AIDS, e verificar a importância da utilização de metodologias educativas que favoreçam a integração dos adolescentes envolvidos no processo, possibilitando a troca de experiências e informações e conscientizando sobre as maneiras de prevenção de DST/AIDS.

A realização desta pesquisa evidencia a necessidade de construção do conhecimento científico da enfermagem com enfoque nas ações educativas desenvolvidas com adolescentes em situação de vulnerabilidade às DST/AIDS. Contribui, ainda, para que as pesquisas da enfermagem que visam à promoção da saúde do adolescente vulnerável às DST/AIDS cumpram a sua finalidade de conduzir a práticas e intervenções efetivas e de qualidade. Isso fará com que as ações de enfermagem direcionadas a essa população sejam implementadas e alcancem resultados efetivos.

Visualizamos que o trabalho em grupo favorece a integração dos sujeitos envolvidos no processo educativo, a troca de experiências e informações, bem como a conscientização a partir do uso de metodologias motivadoras e participativas. Estas sensibilizam o sujeito à mudança de comportamento e, consequentemente, à otimização da qualidade de vida.

Concluímos, a partir dos estudos que intentaram identificar o nível de conhecimento dos adolescentes sobre a temática da investi- gação, que o déficit de informação sobre os aspectos relacionados ao desenvolvimento da sexualidade, algo mais aguçado na adolescência, faz com que esses sujeitos fiquem expostos aos riscos de se infectarem com uma DST/AIDS.

Sugerimos que, além do aprofundamento do tema, o enfermeiro ao desenvolver ações educativas visando à prevenção das DST/ AIDS, crie estratégias de promoção da saúde que levem os adolescentes a conhecer as maneiras de prevenção das DST/AIDS, para que possam desenvolver a sua sexualidade de forma segura.

\section{REFERÊNCIAS}

1. Ministério da Saúde (BR). Boletim Epidemiológico da AIDS 2003 [Internet]. [Brasilia]: Secretaria de Políticas de Saúde. Coordenação Nacional de DST e AIDS (BR); 2004 Março [citado 30 mai 2010]. Disponível em: http://www.aids. gov.br

2. Organização Mundial da Saúde. Relatório de 2007. Um futuro mais seguro: saúde pública global no século XXI [Internet]. Genebra: Organização Mundial da Saúde Geneva; 2007 [citado 1 jun 2010]. 72 p. Disponível em: http://www.portaldasaude.pt

3. Ministério da Saúde (BR). Secretaria Especial de Políticas para as mulheres. Departamento de DST e Aids e da Área Técnica de Saúde da Mulher. Plano Integrado de Enfrentamento da Feminização da Epidemia de AIDS e outras DST. Brasília: Ministério da Saúde, 2007.

4. Morisson-Beedey D, Nelson LE. HIV Prevention interventions in adolescent girls: what is the state of the science? Worldviews Evid Based Nurs. 2004; 1(3): 165-75.

5. Bastable SB. O enfermeiro como educador. $3^{a}$ ed. Porto Alegre: Artmed; 2010. 
6. Orellana A, Paravic T. Enfermería basada en evidencia: barreras y estrategias para su implementación. Cienc. enferm. 2007; XIII(1): 17-24.

7. Whittemore R, Knafl K. The integrative review: methodology. J Adv Nurs. 2005; 52(5): 546-53.

8. Mendes KDS, Silveira RCCP, Galvão CM. Revisão integrativa: método de pesquisa para a incorporação de evidências na saúde e na enfermagem. Texto contexto - enferm. 2008; 17(4): 758-64.

9. Pompeo DA. Diagnóstico de enfermagem náusea em pacientes no período pós-operatório imediato: revisão integrativa da literatura [Dissertação de Mestrado]. [Ribeirão Preto (SP)]: Escola de Enfermagem de Ribeirão Preto da Universidade de São Paulo; 2007. 184 p.

10. Pompeo DA, Rossi LA, Galvão CM. Revisão integrativa: etapa inicial do processo de validação de diagnóstico de enfermagem. Acta paul enferm. 2009; 22(4): 43438.

11. Silveira CS. A pesquisa em enfermagem oncológica no Brasil: uma revisão integrativa [Dissertação de Mestrado]. [Ribeirão Preto (SP)]: Escola de enfermagem de Ribeirão Preto da Universidade de São Paulo; 2005. 116 p.

12. Souza MM, Brunini S, Almeida NAM, Munari DB. Programa educativo sobre sexualidade e DST: relato de experiência com grupo de adolescentes. Rev Bras Enferm. 2007; 60(16): 102-5.

13. Murakami JK, Pitrilli Filho JF; Telles Filho PC. Conversando sobre sexualidade, IST e AIDS com adolescentes pobres. Rev Lat Am Enfermagem. 2007; 15(spe): 8646.

14. Beserra EP, Pinheiro PNC, Barroso MGT. Ação educativa do enfermeiro na prevenção de doenças sexualmente transmissíveis: uma investigação a partir das adolescentes. Esc. Anna Nery. 2008; 12 (3): 522-28.

15. Vieira NFC, Paiva TC, Sherlock MSM.
Sexualidade, DST/AIDS e adolescência: não quero falar, tenho vergonha. DST $\mathrm{j}$ bras doenças sex transm. 2001; 13 (4): 46-51.

16. Koerich MS, Baggio MA, Backes MTS, Backes DS, Carvalho JN, Meirelles BHS, Erdmann AL. Sexualidade, doenças sexualmente transmissíveis e contracepção: atuação da enfermagem com jovens de periferia. Rev enferm UERJ. 2010; 18 (2): 265-71.

17. Dias FLA, Silva KL, Vieira NFC, Pinheiro PNC, Maia CC. Riscos e vulnerabilidades relacionados à sexualidade na adolescência. Rev enferm UERJ. 2010; 18(3): 45661.

18. Marta CB, Francisco MTR, Martins ERC, Clos AC. A Prevenção da AIDS entre Estudantes ao Iniciar o Curso de Graduação em Enfermagem. Rev Enferm UERJ. 2008; 16(4): 557-61.

19. Alves CA, Brandão ER. Vulnerabilidades no Uso de Métodos Contraceptivos entre Adolescentes e Jovens: Interseções entre Políticas Públicas e Atenção a Saúde. Cien Saude Colet. 2009; 14(2): 661-70.

20. Leopardi MT. Metodologia da pesquisa na saúde. Santa Maria: Pallotti; 2001.

21. Barbosa RG, Garcia FCP, Manzato AJ, Martins RA, Vieira FT. Conhecimento sobre DST/AIDS, hepatites e conduta sexual de universitários de São José do Rio Preto, SP. DST j bras doenças sex transm. 2006; 18(4): 224-30.

22. Oliveira SG, Ressel LB. Grupos de adolescentes na prática de enfermagem: um relato de experiência. Cienc Cuid Saude. 2010; 9(1): 144-48.

23. Araújo A, Rocha RL, Armond LC. O grupo de adolescentes na escola: a percepção dos participantes. REME rev min enferm. 2008; 12(2): 207-12.

24. Camilo VMB, Freitas FLS, Cunha VM, Castro RKS, Sherlock MSM, Pinheiro PNC, Vieira NFC. Educação em saúde sobre DST/AIDS com adolescentes de uma escola pública, utilizando a tecnolo- 
gia educacional como instrumento. DST j bras doenças sex transm. 2009; 21(3): $124-28$.

25. Lucchese R, Barros S. A utilização do grupo operativo como método de coleta de dados em pesquisa qualitativa. Rev Eletr Enf [periódico na Internet]. 2007 [citado 1 jun 2010]; 9(3): 796-805. Disponível em: http://www.fen.ufg.br/revista/v9/n3/v9n3a18.htm.
26. Naidoo J, Wills J. Health Promotion: foundations for practice. London: Bailliére; 1994.

27. Chagas NR, Ramos IC, Silva LF, Monteiro ARM, Fialho AVM. Cuidado crítico e criativo: contribuições da educação conscientizadora de Paulo Freire para a enfermagem. Cienc. enferm. 2009; $\mathrm{XV}(2): 35-40$. 